\title{
Experimental Validation of an Efficient Charge Equalization System for Lithium-Ion Batteries
}

\author{
Federico Baronti*, Roberto Roncella*, Roberto Saletti*, Walter Zamboni ${ }^{\S}$ \\ ${ }^{*}$ Dip. di Ingegneria dell'Informazione - Università di Pisa, Italy \\ $\S^{\S}$ Dip. di Ingegneria dell'Informazione, Ingegneria Elettrica e Matematica Applicata, Università degli Studi di Salerno, Italy \\ Email: f.baronti@iet.unipi.it
}

\begin{abstract}
The experimental validation of a Battery Management System (BMS) provided with an innovative high-efficiency active balancing circuit is described in this paper. Charge equalization among the series connected battery cells is achieved with a cell to cell balancing topology. The balancing circuit consists of a switch matrix for the individual access of each cell and a bidirectional DC-DC converter to transfer a controlled amount of charge from a cell to a supercapacitor and back to another cell. The balancing algorithm and the experimental setup are also described. The experiments show the functionality of the balancing circuit that restores a fully balanced battery consisting of eleven $40 \mathrm{~A} \mathrm{~h}$ Lithium-ion cells. For instance, if one of the cells is $17.7 \%$ imbalanced, battery equalization lasts around $25 \mathrm{~h}$ and only costs $1 \%$ of the battery energy. It allows the recovery of the full battery charge, otherwise limited to $82.3 \%$, with an energy saving factor of 6 , if compared with passive balancing. The efficiency of the process, calculated by measuring the energy lost during balancing, is higher than $75 \%$.
\end{abstract}

\section{INTRODUCTION}

A safe and effective use of Lithium-ion batteries requires the adoption of a Battery Management System (BMS) to protect the battery against overcharge and deep discharge and extend its lifetime [1], [2]. The BMS is also asked to provide a charge equalization function, when the battery consists of seriesconnected cells. Differences in the cell capacity, self-discharge rate and operating temperature cause the charge level to vary from cell to cell. This charge imbalance reduces the usable capacity of the battery and thus its lifetime [3]. In fact, the recharge of the battery must be stopped when the cell with the highest charge level reaches the charge cut-off voltage. Thus, the other cells are not fully recharged and the battery is not completely refilled. The cell with the minimum charge level determines in its turn the usable capacity of the battery (i.e., the amount of charge that can be extracted from the battery in the subsequent discharge), as it reaches before the other cells the discharge cut-off voltage.

The simplest approach to equalize the charge among the battery cells is to discharge those with a higher charge level down to the minimum level by means of a shunt-resistor connected in parallel. This is called passive balancing, as the extra energy stored in the cells with higher charge levels is dissipated over the relevant shunt-resistors mounted on the BMS. The maximum usable capacity of the battery is then restored by following the balancing phase with a battery recharge, which indeed leads each cell to be fully charged.
The power dissipated by the balancing circuit has a significant impact on the thermal management of the BMS and decreasing the power dissipated in the passive balancing circuit implies a longer balancing time. The development of a circuit capable of equalizing the battery without dissipating all the extra energy has attracted a lot of research with the aim of improving the trade-off between efficiency, balancing time and complexity [4]-[7]. The most promising approaches are based on a DC/DC converter and a switch matrix that selects one particular cell and connects it to one port of the converter to individually modify its charge. The other port of the converter is typically connected to the battery's terminals, so that energy is transferred from all the cells to the selected one or vice versa (pack to cell or cell to pack topology), according to the direction of the converter [8]-[11].

The balancing time and the energy lost by the battery are related to the efficiency and output current of the converter, but are also proportional to the overall amount of charge that has to be moved to reach the balanced state. As the transfers always occur between all the cells and the selected one in the pack to cell and cell to pack topologies, it may happen that some of the cells are further imbalanced during a transfer. For instance, when the converter is charging the cell with the lowest charge level, all the other cells are discharged, even if their final equalized charge level is higher. Thus, when using the pack to cell and cell to pack balancing topologies, each cell reaches the balanced state by exchanging more charge than the minimum needed. This limitation is overcome by the cell to cell balancing topology, in which energy is transferred from one cell to another selected cell. A serious drawback of this topology is the need for two switch matrices to select the input and output cells of the converter. However, this drawback can be overcome by splitting the cell to cell energy transfer into two phases and using a supercapacitor to temporary store the energy drawn from one cell before delivering it to another. The feasibility of this technique has been demonstrated in [12], showing that the cell to cell energy transfer can be achieved with very high efficiency and affordable complexity.

The aim of this paper is to show the implementation and the experimental validation of a charge equalization system exploiting the technique described in [12] within a BMS for Lithium-ion cells with Nickel Manganese Cobalt (NMC) cathode [13]. The paper is organized as follows. Section II describes the implementation of the charge equalization 


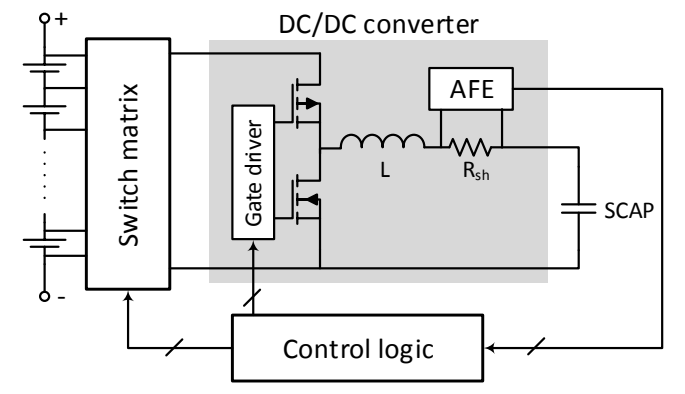

Figure 1. Block diagram of the charge equalization system.

system. The test methodology and the experimental results are presented in Section III and IV, respectively. Finally, some conclusions are drawn in Section V.

\section{Charge EQuAlizATION SYSTEM}

We start by briefly recalling the architecture of the devised cell to cell balancing topology, the block diagram of which is shown in Fig. 1. The Switch matrix block routes the selected cell to one port of the $D C / D C$ converter. A $5 \mathrm{~F}$ supercapacitor (SCAP) is connected to the other port. It is the implemented with $45 \mathrm{~F}$ SCAPs connected in the configuration $2 \mathrm{P} 2 \mathrm{~S}$, so that it can withstand the maximum cell voltage. The converter operates in step-down mode to charge the SCAP from the Source cell. Then, the Sink cell is selected by the switch matrix and the energy, temporary stored in the SCAP, is delivered to the Sink cell by the converter, working in step-up mode. The SCAP charging/discharging current is controlled to remain between two fixed thresholds. These thresholds are set to $0.6 \mathrm{~A}$ and $1 \mathrm{~A}$ by a window comparator implemented in the Analog Front End (AFE), the output of which feeds the Control logic block. The latter controls the converter $n$ - and p-MOSFET switches, to charge/discharge the SCAP between two softwareprogrammable voltage values with an average current of $0.8 \mathrm{~A}$. In the experiments described in the following, the charge and discharge voltage thresholds of the SCAP were set to $0.2 \mathrm{~V}$ and $0.7 \mathrm{~V}$ less than the voltage of the Sink cell, respectively. The control logic also drives the switch matrix according to the balancing algorithm described below. The selection of the switch matrix and converter components has been described in [12]. The reported cell to cell energy transfer efficiency is close to $90 \%$, when the conduction losses in the switch matrix and in the converter are taken into account.

\section{A. Balancing algorithm}

An important aspect of this work is the development of the balancing algorithm that controls the charge equalization system to attain the balanced state. The key idea is to use an iterative procedure, in which each step involves the transfer of a given amount of energy from the cell with the highest charge level to the cell with the lowest one. When the

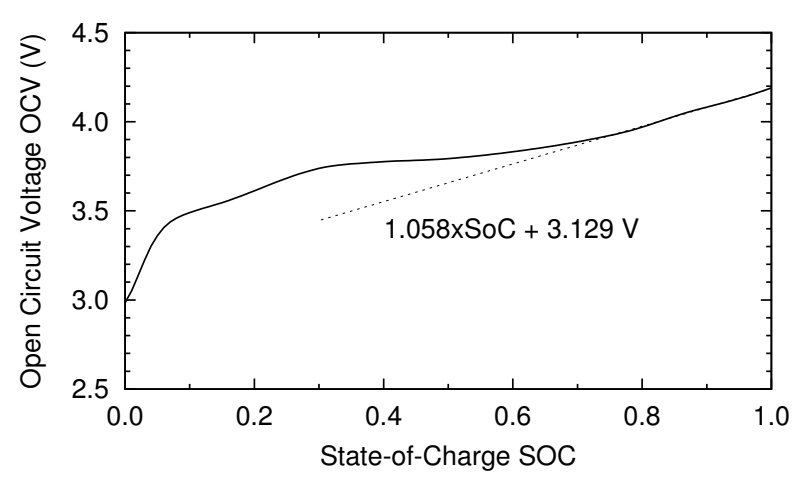

Figure 2. Measured Open Circuit Voltage of a NMC cell.

difference between these two charge levels drops below a desired threshold, the procedure ends. The key point is now the evaluation of the charge levels. Since we are not interested in the absolute values but in the differences among the cell charge levels, we chose to derive the variations of the cell charges from the variations of the cell Open Circuit Voltages (OCVs). As shown in Fig. 2, a $1 \%$ variation of the State of Charge $(S o C)$ (i.e., the ratio of the charge level to its maximum value when the cell is fully charged) corresponds to approximately a $10 \mathrm{mV}$ variation of the $\mathrm{OCV}$, when $\mathrm{SoC}$ is above $80 \%$. Another problem rises from the fact that the measured cell voltage differs from the OCV because of the relaxation voltage [14] due to the battery dynamics. However, we can reasonably suppose that the relaxation voltage term is similar for all the cells, as they all are series connected and subjected to the same charge/discharge operation. Further, the dynamics effects induced by the balancing process, which are different between the two cells involved in the charge transfer, can be neglected if the balancing current is a small fraction of the cell C-rate (i.e., the value of the cell capacity expressed in ampere hour), as it usually happens.

Figure 3 shows the flow diagram of the developed balancing algorithm. Each iteration starts with the measurement of the cell voltages and the identification of their minimum and maximum values. If they differ less than $10 \mathrm{mV}$ the battery is considered balanced (i.e., the maximum $\mathrm{SoC}$ mismatch is less than $1 \%$. Otherwise, the charge equalization system is used to transfer a given amount of energy from the cell with maximum SoC (Source cell) to the cell with the minimum one (Sink cell). The transferred energy quantity is determined by the number $K$ of transfers from the Source cell to the Sink cell performed before passing to the next iteration. The iterations are spaced in time by a $10 \mathrm{~s}$ pause, to allow the expiration of the fast dynamic transients. The choice of $K$ is a trade-off between the balancing resolution and the time overhead due to the $10 \mathrm{~s}$ pause. In the experiments described below, $K$ was set to 5 providing a very fine balancing resolution with the penalty of a longer balancing time. 


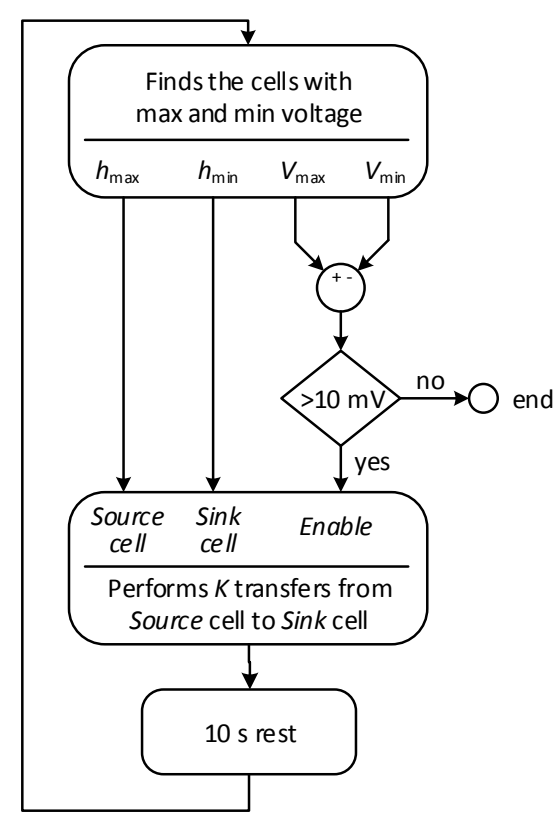

Figure 3. Basic flow diagram of the balancing algorithm.

\section{EXPERIMENTAL SET-UP}

A key point to assess the performance of a balancing circuit lies in the definition of a flexible, reliable and automatic test methodology, which makes it possible to set an arbitrary imbalance condition and then to evaluate the result of the balancing process carried out by the BMS. The designed test methodology consists of the experimental test-bed and the test procedure described below.

\section{A. Test-bed}

The charge equalization was implemented in a BMS for a battery pack of eleven $40 \mathrm{Ah}$ NMC cells. The design of the BMS building blocks is presented in [15] and a simplified schematic view of the BMS is shown in Fig. 4. The voltage and temperature of each cell are measured by the Cell Monitoring Unit (CMU), which communicates with the BMS intelligence through a shared bus. The BMS intelligence incorporates the Control logic of the charge equalization system shown in Fig. 1. The output of the switch matrix is also connected to a SourceMeter (Keithley 2420), so that the charge of the cell selected by the switch matrix can individually be modified with high accuracy. In this way, it is possible to generate a known imbalance condition before activating the charge equalization system. Finally, we can verify the quality of the balanced state achieved by the system by measuring the final charge of each cell using the SourceMeter. The execution of the test is automatically managed by a LabVIEW application, which controls the SourceMeter and communicates with the BMS. Through this link, the LabVIEW application can control

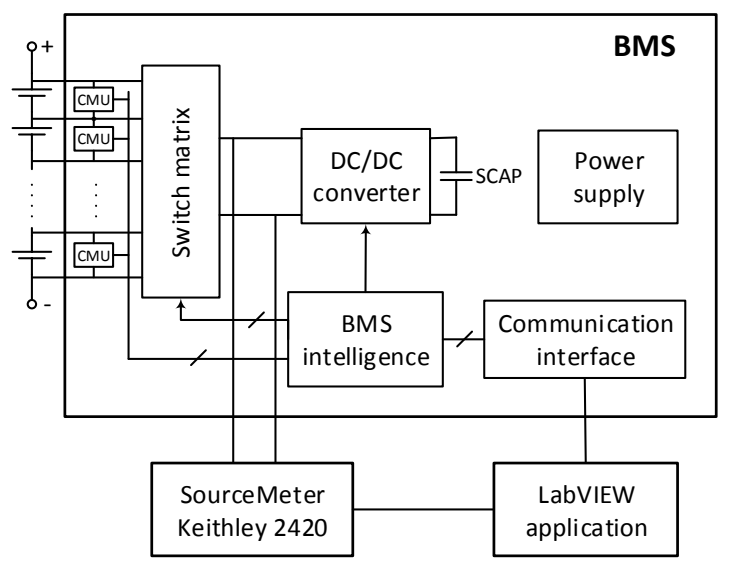

Figure 4. Block diagram of the experimental test-bed.

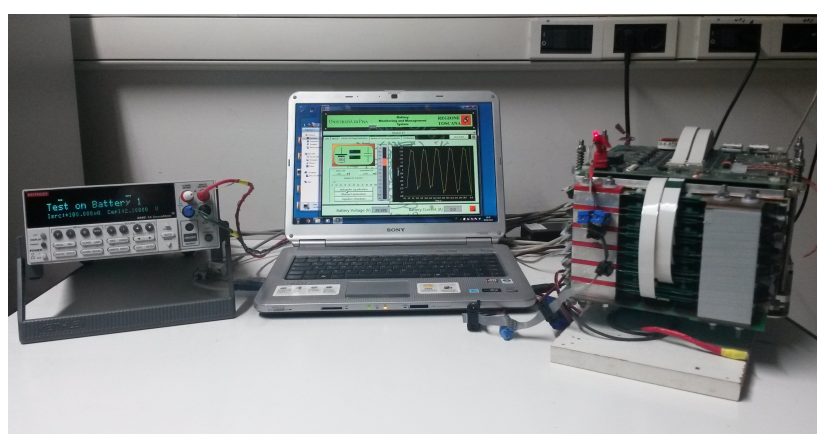

Figure 5. Photograph of the experimental test-bed. Note the battery pack on the right end side.

the switch matrix, so that the leads of the SourceMeter can be connected to any cell of the battery, activate the charge equalization system and log the cell voltages acquired by the BMS during the execution of the test procedure. The latter is detailed in the following.

\section{B. Test procedure}

The test procedure involves the following six steps, where $h \in\{1 \ldots N\}$ with $N=11$ identifies one cell of the battery.

1) Full charge: starting from an arbitrary condition, each cell is individually charged with a Constant Current (CC) of $3 \mathrm{~A}$ up to $4.1 \mathrm{~V}$. Then, the charge proceeds at Constant Voltage (CV) until the current decreases below 0.6 A. This step brings each cell is in a well-defined and reproducible state that we consider the reference starting point $(100 \%$ SoC).

2) Unbalancing: the charge stored in each cell is individually altered in order to generate the desired imbalance state. The charge Qunbal $_{h}$ and Eunbal $_{h}$ drawn from each cell are measured.

3) $1 \mathrm{~h}$ rest: the OCV Vunbal $_{h}$ of each cell is measured at the end of this step. 
4) Equalization: this step is automatically carried out by the BMS. The cell voltages acquired by the BMS are logged every $60 \mathrm{~s}$.

5) $1 \mathrm{~h}$ rest: the OCV $\mathrm{Vbal}_{h}$ of each cell is measured at the end of this step.

6) Full charge: this step is exactly the same as the first one and it is assumed that each cell is brought to the same internal state as at end of the first step. The charge $Q b a l_{h}$ and energy $\mathrm{Ebal}_{h}$ delivered to each cell are measured.

The final step provides a direct measure of the quality of the balancing process performed at step 4 . The refilling charge value $\mathrm{Qbal}_{h}$ should be the same for any cell in an ideally balanced battery, as all the cells are equidistant from the full charge state. Thus, charging the battery from its terminals after the equalization step allows all the cells to be fully recharged. As a consequence, the maximum usable capacity of the battery reaches the ideal value $Q_{\max }$. Instead, different $Q b a l_{h}$ values show a residual imbalance after the equalization step. In this case, the battery cannot be used in full, as the usable capacity is reduced by the difference between the maximum and minimum values of $Q_{b a l}$.

This test structure makes it possible to measure the energy $E_{\text {loss }}$ lost by the battery to recover the balanced condition. In fact, $E_{\text {loss }}$ can be computed from Eunbal $_{h}$ and Ebal $_{h}$ according to (1).

$$
E_{\mathrm{loss}}=\sum_{h=1}^{N} E_{b a l_{h}}-\text { Eunbal }_{h}
$$

Assuming that each cell stores the same amount of energy after a Full charge (steps 1 and 6), Ebal $l_{h}-E_{\text {Enbal }}$ is the variation of the energy stored in the $h$-th cell during the equalization step.

One of the main sources that contributes to $E_{\text {loss }}$ is the DC/DC converter inefficiency. Assuming that the cells are sorted in descending order of the stored charge, the balancing algorithm adopted extracts energy only from the $M$ more charged cells delivering it to the remaining $N-M$ cells, where $M$ depends on the actual charge distribution among the cells. If $M$ is known, the converter efficiency $\eta$ can be computed as the energy delivered to the $N-M$ less charged cells divided by the energy extracted from the $M$ more charged ones, as shown in (2).

$$
\eta=\frac{\sum_{h=M+1}^{N} \text { Eunbal }_{h}-\text { Ebal }_{h}}{\sum_{h=1}^{M} \text { Ebal }_{h}-\text { Eunbal }_{h}}
$$

\section{EXPERIMENTAL RESULTS}

First experiments allowed us to verify the BMS capability to transfer energy between the cells of the battery and to achieve cell balancing. Then, the balancing circuit performance was investigated. To this end, it is important to start with the application of imbalance states, which make it possible to extract the features of the balancing circuit from the results of the balancing process. The imbalance state is specified by the set of individual state-of-charge (SoCunbal) values of the cells,

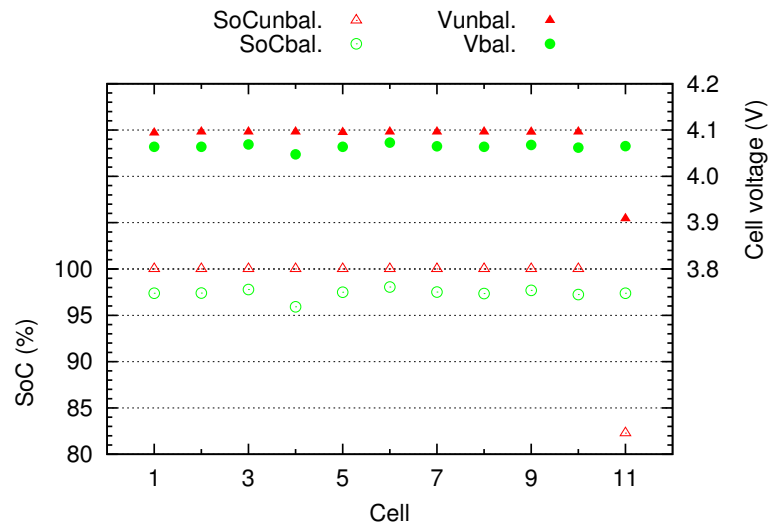

Figure 6. State-of-Charge (SoCunbal, SoCbal) and open circuit voltage (Vunbal, Vbal) of the battery cells, respectively, before and after the balancing process.

as generated after steps 2 and 3 of the test procedure described in Section III-B, according to the following relationship

$$
\text { SoCunbal }_{h}=1-\frac{\text { Qunbal }_{h}}{Q_{\max }}
$$

where $Q_{\max }$ is assumed to be the same for all the 11 cells and equal to their nominal capacity, i.e., $40 \mathrm{~A}$ h. This assumption was verified with a complete charge-discharge cycle for each cell.

Let us now report and discuss the equalization experiment performed after a significant $S o C$ imbalance state. As an example, we considered the condition shown in (4), where all the cells are fully charged apart from the $11^{\text {th }}$ cell characterized by a $S o C 17.7 \%$ lower than the others.

$$
\text { SoCunbal }_{h}=\left\{\begin{aligned}
100 \%, & h \in\{1 \ldots 10\} \\
82.3 \%, & h=11
\end{aligned}\right.
$$

This imbalance state may occur when one cell has an anomalous higher self-discharge rate than the other cells, which causes a significant $\mathrm{SoC}$ mismatch (the difference between the maximum and minimum $\mathrm{SoC}$ values) over time. This turns in a strong reduction of the usable capacity of the battery, unacceptable in many applications if no balancing is applied.

Figure 6 shows the $S o C$ and OCV of each battery cell before and after the execution of the balancing process according to the test procedure described in Section III-B and the SoC imbalance of (4). It is worth noting that the $S o C$ values after balancing $\mathrm{SoCbal}_{h}$, calculated according to the following equation by measuring the refilling charge $Q_{b a l_{h}}$

$$
\text { SoCbal } l_{h}=1-\frac{Q b a l_{h}}{Q_{\max }}
$$

are all very close to each other and their values are close to $100 \%$. The experiment shows the functionality of the balancing system that significantly reduces the $S o C$ imbalance, as charge is "equally" transferred from the cells $1-10$ to the cell 11. In more detail, the initial $S o C$ mismatch of around 


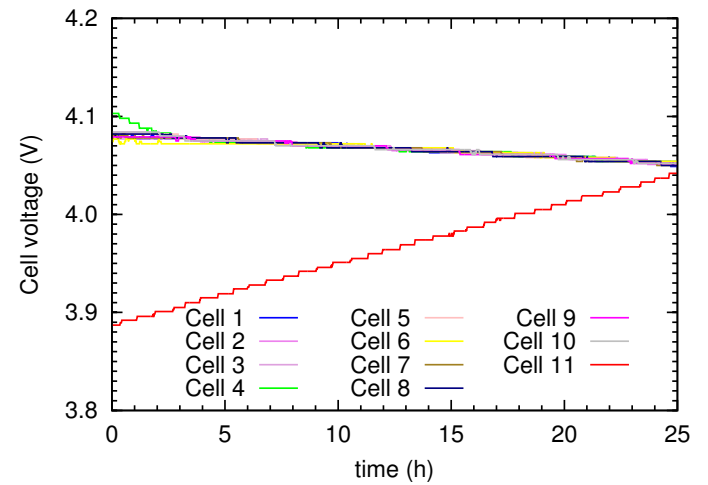

Figure 7. Battery cell voltages as a function of time during balancing.

$18 \%$ is reduced to $2 \%$, with a consequent $16 \%$ increase of the usable battery capacity. The battery has successfully been balanced.

The result achieved is outstanding, but the residual $S o C$ mismatch is slightly higher than the $1 \%$ expected from the balancing algorithm described in Section II-A. As visible in Fig. 6, $\mathrm{SoCbal}_{4}$ is slightly below the $\mathrm{SoC}$ values of the other cells, which instead lie in just a $0.8 \%$ SoC range. Further, we observe that the OCV values measured at the end of steps 3 and 5 by the SourceMeter very well agree with the correspondent $S o C$ values and the SoC-OCV relationship shown in Fig. 2.

In order to investigate the different behavior of the cell 4 , we analyzed the voltages of the cells during the equalization step, as measured and logged by the BMS. They are shown in Fig. 7. It is clearly visible that the voltage of cell 4 measured by the BMS at the beginning of the balancing process is higher than the value of the other fully charged cells, which show almost identical values in good accordance with those reported in Fig. 6, as measured by the external SourceMeter. The conclusion is that the discrepancy is due to a problem in the CMU connected to cell 4, which causes a positive offset of about $25 \mathrm{mV}$ in its voltage reading ( $25 \mathrm{mV}$ approximately corresponds to a $2 \% \mathrm{SoC}$ variation). Thus, the balancing algorithm, which relies on the inaccurate voltage measurement of cell 4, draws more charge from this cell than from the other cells in order to bring all the cell voltages in a $10 \mathrm{mV}$ range at the end of the balancing process, as required by the balancing algorithm described in Fig. 3.

Figure 7 also shows that the balancing process lasts approximately $25 \mathrm{~h}$. This value is proportional to the charge that has to be moved to reach the balanced state starting from the initial imbalance condition. Given the $S o C$ imbalance of (4), the charge to be transferred to the cell 11 is estimated by 10 Qunbal $_{11} / 11=0.16 Q_{\max }=6.4 \mathrm{~A} \mathrm{~h}$, without considering the converter efficiency. Thus, the net balancing current is approximately $0.26 \mathrm{~A}$. This value is less than half of the SCAP charging and discharging current (i.e., 0.8 A), as it includes all the time overheads due to the pauses after each block of 5 transfers, and to the commutation times of the switch matrix after each charge/discharge of the supercapacitor. It should be noted that in a real application the battery balancing can start whenever the maximum $\mathrm{SoC}$ mismatch reaches a given thresholds (typically a few percents) well below the $18 \%$ value used in our experiment. Therefore, the balancing time will be significantly smaller than the one experimented in our case.

\section{A. Energy losses during balancing}

Another key aspect to explore for the validation of the balancing technique is the evaluation of the energy lost by the battery during the balancing process. Substituting in (1) for $E_{\text {unbal }}$ and $E_{b a l}$ the values measured at the steps 2 and 6 respectively, we obtain $E_{\text {loss }}=19.1 \mathrm{Wh}$. This value is around $1 \%$ of the maximum energy $E_{\max }$ that can be stored in the battery, estimated as $E_{\max }=11 \times 3.7 \times \mathrm{Q}_{\max }=1628 \mathrm{Wh}$. This means that in our case study the battery is balanced again (gaining $16 \%$ of the usable capacity) at the expense of only $1 \%$ of the energy. Neglecting the residual $S o C$ imbalance, the usable energy of the battery (which was $83 \%$ of $E_{\max }$ before balancing) can be restored to $E_{\max }$ by delivering to the battery the energy $E_{\text {loss }}+$ Eunbal $_{11}=0.029 E_{\max }$ through a recharge step that follows balancing. It is now interesting to compare this result with that achievable with passive balancing. In that case, the energy required to restore the battery to $E_{\max }$ would be 11 Eunbal $_{11}=0.19 E_{\max }$, as the balanced state is reached by discharging all the cells $1-10$ to the level of cell 11 . This value is around 6.5 times higher than the one found for the implemented cell to cell topology, and shows the maximum energy gain that this active technique allows us to reach.

As the cells 1-10 are discharged only, whereas the cell 11 is charged only during our active balancing (as shown in Figure 7), it is possible to evaluate the efficiency of the converter from (2) by substituting $M=10, N=11$, and for $E_{\text {unbal }}$ and $\mathrm{Ebal}_{h}$ the values measured at step 2 and 6 , respectively. This leads to $\eta=55 \%$. Such a value is significantly lower than the expected stand-alone efficiency of the converter. In fact, the energy lost by the battery includes that dissipated by the balancing circuit, but also the energy consumed by the BMS electronics. Thus, a better estimation of $\eta$ can be obtained by the following relationship,

$$
\eta=\frac{\sum_{h=M+1}^{N}\left(\text { Eunbal }_{h}-E_{b b a l_{h}}+E_{\mathrm{CC} / N}\right)}{\sum_{h=1}^{M}\left(\text { Ebal }_{h}-\text { Eunbal }_{h}-E_{\mathrm{CC} / N}\right)}
$$

where $E_{\mathrm{CC}}$ is the energy consumed by the BMS during the balancing time. $E_{\mathrm{CC}}$ can be evaluated by multiplying the measured average power consumption of the BMS (approximately $0.44 \mathrm{~W}$ ) for the balancing time $(25 \mathrm{~h})$ and is equal to $11 \mathrm{Wh}$. Substituting this value in (6), we obtain $\eta=75 \%$, a valuable result, even if lower than the actual converter efficiency. Finally, we note that the methodology used to derive the converter efficiency is affected by some uncertainties, which cause $\eta$ to be underestimated. In particular, (6) should also include the intrinsic losses of the battery due to its ohmic resistance and not ideal coulombic efficiency. Another 


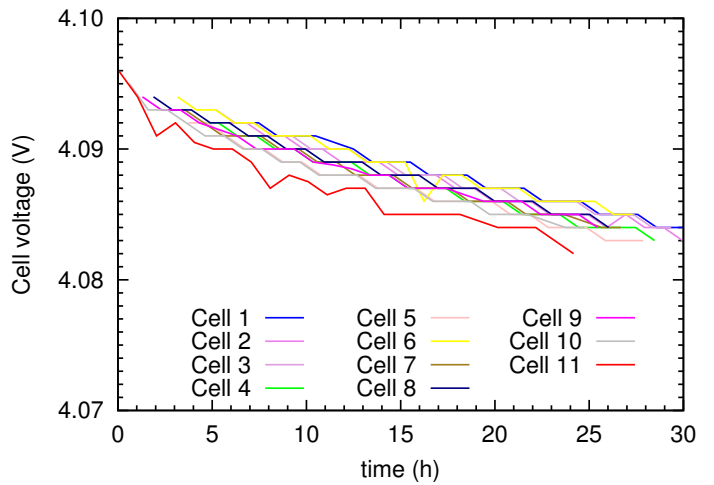

Figure 8 . Voltage of the battery cells measured during a $24 \mathrm{~h}$ rest time after individual full charge.

uncertainty is related to the relaxation of the cell voltage during the balancing experiment. This aspect was investigated by performing another test consisting of the following steps: a full charge, a $24 \mathrm{~h}$ rest and another full charge. Figure 8 shows the behavior of the cell voltages measured during the $24 \mathrm{~h}$ rest, as a function of time, counted from the end of the full charge. If we now consider the overall energy delivered to all the cells during the second full charge, we obtain around $18.6 \mathrm{~W} \mathrm{~h}$, which is appreciably higher than the energy consumed by the BMS. This means that the cells, after a $24 \mathrm{~h}$ relaxation, can accept a value of additional energy, which is not negligible in the converter efficiency calculation. In fact, this relaxation contribution is included into Eunbal $h$ and considered as an additional loss of the balancing in (6), causing a significant underestimate of $\eta$.

\section{Conclusions}

This paper has shown and discussed some experimental results carried out to validate a BMS provided with an innovative high-efficiency active balancing circuit. The circuit implements a cell to cell balancing topology in which energy is transferred from a less charged cell to a more charged one, after being temporarily stored in a supercapacitor. A balancing algorithm has been developed and implemented in the BMS. In order to validate the charge equalization system, an automatic test methodology has been implemented. The experiments first show the functionality of the balancing circuit that restores a fully balanced battery consisting of eleven $40 \mathrm{Ah}$ NMC cells, where one of the cells shows an imbalance around $17.7 \%$. The battery equalization lasts around $25 \mathrm{~h}$, only costs $1 \%$ of the battery energy and allows the recovery of the full battery charge, otherwise limited to a $82.3 \%$ utilization. The comparison of this active balancing solution with the passive balancing technique shows that we achieved an energy saving of a factor larger than 6. Finally, the efficiency of the active balancing process is calculated, by measuring the loss in energy occurred during the balancing process.
The efficiency is well over the $75 \%$ value measured only considering the consumption of the BMS electronics and neglecting the intrinsic losses in the battery and the uncertainty of the measurement method.

\section{ACKNOWLEDGEMENT}

The authors would like to acknowledge the major contribution of Dr. Gabriele Fantechi to this work.

\section{REFERENCES}

[1] H. Rahimi-Eichi, U. Ojha, F. Baronti, and M.-Y. Chow, "Battery Management System: An Overview of Its Application in the Smart Grid and Electric Vehicles," IEEE Ind. Electron. Mag., vol. 7, no. 2, pp. 4-16, Jun. 2013.

[2] L. Lu, X. Han, J. Li, J. Hua, and M. Ouyang, "A review on the key issues for lithium-ion battery management in electric vehicles," $J$. Power Sources, vol. 226, pp. 272-288, Mar. 2013.

[3] L. Zhong, C. Zhang, Y. He, and Z. Chen, "A method for the estimation of the battery pack state of charge based on in-pack cells uniformity analysis," Appl. Energy, vol. 113, no. 0, pp. 558-564, Jan. 2014.

[4] A. Baughman and M. Ferdowsi, "Double-Tiered Switched-Capacitor Battery Charge Equalization Technique," IEEE Trans. Ind. Electron., vol. 55, no. 6, pp. 2277-2285, Jun. 2008.

[5] P. A. Cassani and S. S. Williamson, "Design, Testing, and Validation of a Simplified Control Scheme for a Novel Plug-In Hybrid Electric Vehicle Battery Cell Equalizer," IEEE Trans. Ind. Electron., vol. 57, no. 12 , pp. 3956-3962, Dec. 2010.

[6] M. Daowd, N. Omar, P. Van Den Bossche, and J. Van Mierlo, "Passive and active battery balancing comparison based on MATLAB simulation," in 2011 IEEE Veh. Power Propuls. Conf. IEEE, Sep. 2011, pp. 1-7.

[7] J. Gallardo-Lozano, E. Romero-Cadaval, M. I. Milanes-Montero, and M. A. Guerrero-Martinez, "Battery equalization active methods," $J$. Power Sources, vol. 246, pp. 934-949, Jan. 2014.

[8] C.-H. Kim, M.-Y. Kim, and G.-W. Moon, "A Modularized Charge Equalizer Using a Battery Monitoring IC for Series-Connected Li-Ion Battery Strings in Electric Vehicles," IEEE Trans. Power Electron., vol. 28, no. 8, pp. 3779-3787, Aug. 2013.

[9] F. Baronti, C. Bernardeschi, L. Cassano, A. Domenici, R. Roncella, and R. Saletti, "Design and Safety Verification of a Distributed Charge Equalizer for Modular Li-ion Batteries," IEEE Trans. Ind. Informatics, vol. PP, no. 99, pp. 1-1, 2014.

[10] A. M. Imtiaz and F. H. Khan, “"'Time Shared Flyback” Converter Based Regenerative Cell Balancing Technique for Series Connected Li-Ion Battery Strings," IEEE Trans. Power Electron., vol. 28, no. 12, pp. 5960-5975, Dec. 2013.

[11] T. A. Stuart and W. Zhu, "Modularized battery management for large lithium ion cells," J. Power Sources, vol. 196, no. 1, pp. 458-464, Jan. 2011.

[12] F. Baronti, G. Fantechi, R. Roncella, and R. Saletti, "High-Efficiency Digitally Controlled Charge Equalizer for Series-Connected Cells Based on Switching Converter and Super-Capacitor," IEEE Trans. Ind. Informatics, vol. 9, no. 2, pp. 1139-1147, May 2013.

[13] M. S. Whittingham, "History, Evolution, and Future Status of Energy Storage," Proc. IEEE, vol. 100, no. Special Centennial Issue, pp. 1518-1534, May 2012.

[14] F. Baronti, W. Zamboni, N. Femia, H. Rahimi-Eichi, R. Roncella, S. Rosi, R. Saletti, and M.-Y. Chow, "Parameter identification of Li-Po batteries in electric vehicles: A comparative study," in 2013 IEEE Int. Symp. Ind. Electron. IEEE, May 2013, pp. 1-7.

[15] F. Baronti, G. Fantechi, R. Roncella, R. Saletti, and P. Terreni, "Hardware building blocks of a hierarchical battery management system for a fuel cell HEV," in IECON 2012 - 38th Annu. Conf. IEEE Ind. Electron. Soc. IEEE, Oct. 2012, pp. 4041-4047. 